\title{
Coordinate-space Faddeev-Hahn-type approach to three-body charge-transfer reactions involving exotic particles
}

\author{
Renat A. Sultanov and Sadhan K. Adhikari \\ Instituto de Física Teórica, Universidade Estadual Paulista, 01405-900 São Paulo, São Paulo, Brazil
}

(Received 22 February 1999; published 12 January 2000)

\begin{abstract}
Low-energy muon-transfer cross sections and rates in collisions of muonic atoms with hydrogen isotopes are calculated using a six-state close-coupling approximation to coordinate-space Faddeev-Hahn-type equations. In the muonic case satisfactory results are obtained for all hydrogen isotopes and the experimentaly observed strong isotopic dependence of transfer rates is also reproduced. A comparison with results of other theoretical and available experimental works is presented. The present model also leads to good transfer cross sections in the well-understood problem of antihydrogen formation in antiproton-positronium collision.
\end{abstract}

PACS number(s): 34.70.+e, 36.10.Dr

\section{INTRODUCTION}

Charge-transfer reactions involving few particles in atomic physics are very challenging and interesting from both theoretical and experimental points of view and we study here the problem of charge transfer in some atomic reactions involving exotic particles. Specifically, we study muon transfer in $\mathrm{D}-\mathrm{H}_{\mu}, \mathrm{T}-\mathrm{H}_{\mu}$, and $\mathrm{T}-\mathrm{D}_{\mu}$ systems where the suffix $\mu$ denotes a muonic atom with the electron replaced by a muon $\left(\mu^{-}\right)$. We also study the problem of antihydrohen $(\overline{\mathrm{H}})$ formation in antiproton-positronium collision with the positronium (Ps) in an initial $1 s$ state.

On the theoretical side, in these transfer reactions one needs to consider rearrangement of a charged particle. Because of the Coulomb interaction one needs a careful treatment of the dynamics for a correct description. If one can identify the basic dynamical ingredients necessary for a satisfactory description of these processes involving a small number of particles, such a study will help us to formulate models in more complex situations. On the experimental side, the present study involving muon and positron transfer is of current interest in the muon-catalyzed fusion cycle [1-6] and in the formation and study of the antihydrogen atom $[7,8]$.

Although there are some experimental measurements and several theoretical investigations of these processes, there still remain discrepancies among various studies. Here we use a different theoretical approach based on a detailed fewbody dynamical consideration for a careful reinvestigation of these three-body charge-transfer reactions. Traditionally, such problems are investigated by a tractable approximation scheme in the Schrödinger framework, without explicitly considering a few-body dynamical equation. In addition to variational calculations, these schemes include closecoupling, hyperspherical, and adiabatic approximations. Here we would like to point out that the processes of muonic transfer reactions and antihydrogen formation are three-body Coulombic rearrangement collisions. Consequently, it seems reasonable that, in addition to approximations based on the Schrödinger equation, a detailed few-body consideration is useful. In what follows we develop a method, which is based on detailed few-body equations rather than the effective po- tential treatment employed in alternative investigations.

For the three-charged-particle system, say TD $\mu$, only two asymptotic configurations are possible, i.e., (D $\mu) \mathrm{T}$ and $(\mathrm{T} \mu) \mathrm{D}$. This suggests to write down a set of two coupled equations for components $\Psi_{1}$ and $\Psi_{2}$ of the wave function $\Psi=\Psi_{1}+\Psi_{2}[9,10]$, with each component carrying the asymptotic boundary condition for a specific configuration. One such equation with two components for the threeparticle system was first written by Hahn [9], following the most general decomposition of the three-body wave function into three components suggested by Faddeev [11], and is usually referred to as the Faddeev-Hahn equation [12]. We solve the integro-differential form of this equation by a sixstate close-coupling approximation scheme that consists in expanding the wave-function components $\Psi_{1}$ and $\Psi_{2}$ in terms of eigenfunctions of subsystem Hamiltonians in initial and final channels, respectively. The resultant coupled equation is then projected on the expansion functions. After a partial-wave projection this leads to a set of one-dimensional coupled equations for the expansion coefficients, which is solved numerically.

Recently, there have been considerable theoretical and experimental interests in the study of the muon-transfer reactions between hydrogen isotopes in the muon-catalyzed fusion cycle

$$
\begin{gathered}
\mathrm{D}+\mathrm{H}_{\mu} \rightarrow \mathrm{D}_{\mu}+\mathrm{H}, \\
\mathrm{T}+\mathrm{H}_{\mu} \rightarrow \mathrm{T}_{\mu}+\mathrm{H}, \\
\mathrm{T}+\mathrm{D}_{\mu} \rightarrow \mathrm{T}_{\mu}+\mathrm{D} .
\end{gathered}
$$

The measurements for the transfer rates

$$
\lambda_{\mathrm{tr}}=\sigma_{\mathrm{tr}} \mathrm{v} N_{0}
$$

with $\sigma_{\text {tr }}$ being the transfer cross section, $\mathrm{v}$ the relative velocity of the incident particles, and $N_{0}=4.25 \times 10^{22} \mathrm{~cm}^{-3}$ the liquid hydrogen density, are listed in Table I together with 
TABLE I. Experimental and theoretical results for the muonic transfer rates $\lambda_{\mathrm{tr}} / 10^{8} \mathrm{~s}^{-1}$ given for low energies $(E<0.1 \mathrm{eV})$.

\begin{tabular}{lrll}
\hline \hline Reaction & \multicolumn{1}{c}{ Experiment } & \multicolumn{2}{c}{ Theory } \\
\hline $\mathrm{D}+\mathrm{H}_{\mu} \rightarrow \mathrm{D}_{\mu}+\mathrm{H}$ & $95 \pm 34[13]$ & $140[20]$ & $159[21]$ \\
& $143 \pm 13[14]$ & $133^{\mathrm{a}}$ & \\
& $84 \pm 13[15]$ & & \\
$\mathrm{T}+\mathrm{H}_{\mu} \rightarrow \mathrm{T}_{\mu}+\mathrm{H}$ & $58.6 \pm 10[16]$ & $55[20]$ & $71.7[21]$ \\
& & $61^{\mathrm{a}}$ & \\
$\mathrm{T}+\mathrm{D}_{\mu} \rightarrow \mathrm{T}_{\mu}+\mathrm{D}$ & $2.9 \pm 0.4[17]$ & $3.5[20]$ & $2.26[21]$ \\
& $2.8 \pm 0.3[18]$ & $1.5^{\mathrm{b}}[22]$ & $2.8[23]$ \\
& $2.8 \pm 0.5[19]$ & $2.39^{\mathrm{b}}[24]$ & $0.93^{\mathrm{b}}[25]$ \\
& $3.5 \pm 0.5[19]$ & $2.3^{\mathrm{a}}$ & \\
\hline \hline
\end{tabular}

${ }^{\mathrm{a}}$ Present results.

${ }^{\mathrm{b}}$ Rates reproduced from cross sections.

recent theoretical calculations. One can see differences between various experimental data [13-19] and theoretical results $[20-25]$.

One of the most attractive reactions for $\overline{\mathrm{H}}$ formation is the three-body positron-transfer process

$$
\bar{p}+\mathrm{Ps} \rightarrow \overline{\mathrm{H}}+e^{-} \text {. }
$$

Although no experimental cross sections are available, this process is being used at CERN for the production and study of antihydrogen. A number of calculations have recently been carried out to calculate the cross section of reaction (3) as a function of the incident Ps energy. The calculations were performed by different methods; for instance, with hyperspherical coupled-channel expansions [26] and closecoupling approximations (CCA's) [27]. As an additional test of the present method, calculations for the $S$-wave antihydrogen formation (3) at low energies are also performed.

In Sec. II we develop the formalism. The results obtained for reactions (1) and (3) are given in Sec. III. Finally, we present some concluding remarks in Sec. IV.

\section{THEORETICAL FORMULATION}

Let us take the system of units to be $e=\hbar=m_{\mu}=1$ and denote, say, $\mathrm{T}$ by $1, \mathrm{D}$ by 2 , and muon by 3 . Below the three-body breakup threshold, the following two-cluster asymptotic configurations are possible in the system (123): (23) -1 and (13)-2. These configurations, denoted simply by 1 and 2 , respectively, are determined by the Jacobi coordinates $\left(\vec{r}_{j 3}, \vec{\rho}_{k}\right)$

$\vec{r}_{j 3}=\vec{r}_{3}-\vec{r}_{j}, \quad \vec{\rho}_{k}=\left(\vec{r}_{3}+m_{j} \vec{r}_{j}\right) /\left(1+m_{j}\right)-\vec{r}_{k}, \quad j \neq k=1,2$,

$\vec{r}_{j}, \quad m_{j}$ being coordinates and masses of the particles $j$ $=1,2,3$, respectively.

Let us introduce the total three-body wave function as a sum of two components

$$
\Psi\left(\vec{r}_{1}, \vec{r}_{2}, \vec{r}_{3}\right)=\Psi_{1}\left(\vec{r}_{23}, \vec{\rho}_{1}\right)+\Psi_{2}\left(\vec{r}_{13}, \vec{\rho}_{2}\right)
$$

where $\Psi_{1}\left(\vec{r}_{23}, \vec{\rho}_{1}\right)$ is quadratically integrable over the variable $\vec{r}_{23}$, and $\Psi_{2}\left(\vec{r}_{13}, \vec{\rho}_{2}\right)$ over the variable $\vec{r}_{13}$. To define $\Psi_{l}(l=1,2)$ a set of two coupled equations can be written as

$$
\begin{aligned}
& \left(E-H_{0}-V_{23}\right) \Psi_{1}\left(\vec{r}_{23}, \vec{\rho}_{1}\right)=\left(V_{23}+V_{12}\right) \Psi_{2}\left(\vec{r}_{13}, \vec{\rho}_{2}\right), \\
& \left(E-H_{0}-V_{13}\right) \Psi_{2}\left(\vec{r}_{13}, \vec{\rho}_{2}\right)=\left(V_{13}+V_{12}\right) \Psi_{1}\left(\vec{r}_{23}, \vec{\rho}_{1}\right),
\end{aligned}
$$

where $E$ is the center-of-mass energy, $H_{0}$ is the total kinetic energy operator, and $V_{i j}\left(r_{i j}\right)$ are pair-interaction potentials $(i \neq j=1,2,3)$. Equations (6) satisfy the Schrödinger equation exactly, and for energies below the three-body breakup threshold they possess the same advantages as the Faddeev equations, since they are formulated for the wave-function components with correct physical asymptotes.

In the general case a component of the three-body wave function has the asymptotic form that includes all open channels: elastic/inelastic, transfer, and breakup. In this case each component of the total wave function carries a specific asymptotic behavior. The component $\Psi_{1}$ carries the asymptotic behavior in elastic and inelastic channels:

$$
\begin{aligned}
& \Psi_{1}\left(\vec{r}_{23}, \vec{\rho}_{1}\right) \widetilde{\rho_{1} \rightarrow+\infty} e^{i k_{1}^{(1)} z} \varphi_{1}\left(\vec{r}_{23}\right) \\
& +\sum_{n} A_{n}^{\mathrm{e} / / \mathrm{in}}\left(\Omega_{\rho_{1}}\right) e^{i k_{n}^{(1)} \rho_{1} / \rho_{1}} \varphi_{n}\left(\vec{r}_{23}\right)
\end{aligned}
$$

The component $\Psi_{2}$ carries the asymptotic behavior in the transfer channels:

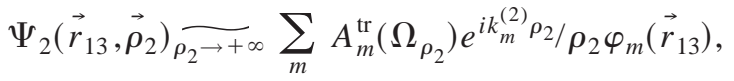

where $e^{i k_{1}^{(1)} z} \varphi_{1}\left(\vec{r}_{23}\right)$ is the incident wave, $\varphi_{n}\left(\vec{r}_{j 3}\right)$ the $n$th excited bound-state wave function of pair $(j 3)$, and $k_{n}^{(i)}$ $=\sqrt{2 M_{i}\left(E-E_{n}^{(j)}\right)}$ with $M_{i}^{-1}=m_{i}^{-1}+\left(1+m_{j}\right)^{-1}$. Here $E_{n}^{(j)}$ is the binding energy of $(j 3), i \neq j=1,2$, and $A^{\mathrm{el} / \mathrm{in}}\left(\Omega_{\rho_{1}}\right)$ and $A^{\operatorname{tr}}\left(\Omega_{\rho_{2}}\right)$ are the scattering amplitudes in the elastic/inelastic and transfer channels. This approach simplifies the solution procedure and simultaneously provides a correct asymptotic behavior for the solution below the three-body breakup threshold.

Let us write Eqs. (6) in terms of the adopted notations

$$
\begin{gathered}
{\left[E+\frac{\nabla_{\vec{\rho}_{k}}^{2}}{2 M_{k}}+\frac{\nabla_{\vec{r}_{j 3}}^{2}}{2 \mu_{j}}-V_{j 3}\right] \Psi_{k}\left(\vec{r}_{j 3}, \vec{\rho}_{k}\right)} \\
=\left(V_{j 3}+V_{j k}\right) \Psi_{j}\left(\vec{r}_{k 3}, \vec{\rho}_{j}\right) .
\end{gathered}
$$

Here $j \neq k=1,2$ and $M_{k}^{-1}=m_{k}^{-1}+\left(1+m_{j}\right)^{-1}, \quad \mu_{j}^{-1}=1$ $+m_{j}^{-1}$.

For solving Eq. (9) we expand the wave-function components in terms of bound states in initial and final channels, and project this equation on these bound states. This prescription is similar to that adopted in the close-coupling approximation. Specifically, we use the following partial-wave expansion: 


$$
\Psi_{k}\left(\vec{r}_{j 3}, \vec{\rho}_{k}\right)=\sum_{L M \lambda l} \Phi_{L M \lambda l}^{(k)}\left(\rho_{k}, r_{j 3}\right)\left\{Y_{\lambda}\left(\hat{\rho}_{k}\right) \otimes Y_{l}\left(\hat{r}_{j 3}\right)\right\}_{L M},
$$

$$
\left\{Y_{\lambda}\left(\hat{\boldsymbol{\rho}}_{k}\right) \otimes Y_{l}\left(\hat{r}_{j 3}\right)\right\}_{L M}=\sum_{m^{\prime} m} C_{\lambda m^{\prime} l m}^{L M} Y_{\lambda m^{\prime}}\left(\hat{\rho}_{k}\right) Y_{l m}\left(\hat{r}_{j 3}\right),
$$

where $C$ 's are the Clebsch-Gordon coefficients, $Y$ 's are the usual spherical harmonics, and $L, \lambda, l$ and $M, m^{\prime}, m$ are the appropriate angular-momentum variables and their projections. Next we make the following close-coupling-type approximation for the radial part in terms of the bound-state wave functions in the initial and final channels:

$$
\Phi_{L M \lambda l}^{(k)}\left(\rho_{k}, r_{j 3}\right) \approx \frac{1}{\rho_{k}} \sum_{n} f_{n l \lambda}^{(k) L M}\left(\rho_{k}\right) R_{n l}^{(k)}\left(r_{j 3}\right),
$$

where radial components of the bound-state wave functions $R_{n l}^{(k)}\left(r_{j 3}\right)$ satisfy

$$
\begin{aligned}
& \left\{E_{n}^{(k)}+\frac{1}{2 \mu_{j} r_{j 3}^{2}}\left[\frac{\partial}{\partial r_{j 3}}\left(r_{j 3}^{2} \frac{\partial}{\partial r_{j 3}}\right)-l(l+1)\right]-V_{j 3}\right\} \\
& \times R_{n l}^{(k)}\left(r_{j 3}\right)=0 .
\end{aligned}
$$

Then we substitute Eqs. (10)-(12) into Eq. (9), multiply the resultant equation by the appropriate biharmonic functions and the corresponding radial functions $R_{n l}^{(k)}\left(r_{j 3}\right)$, and integrate over the corresponding angular coordinates of the vectors $\vec{r}_{j 3}$ and $\vec{\rho}_{k}$. Then we obtain a set of integral differential equations for the unknown functions $f_{n l \lambda}^{(k)}\left(\rho_{k}\right)$,

$$
\begin{aligned}
& 2 M_{k}\left(E-E_{n}^{(j)}\right) f_{\alpha}^{(k)}\left(\rho_{k}\right)+\left\{\frac{\partial^{2}}{\partial \rho_{k}^{2}}-\frac{\lambda(\lambda+1)}{\rho_{k}^{2}}\right\} f_{\alpha}^{(k)}\left(\rho_{k}\right) \\
&=2 M_{k} \sum_{\alpha^{\prime}} \int_{0}^{\infty} d r_{j 3} r_{j 3}^{2} \int d \hat{r}_{j 3} \int d \hat{\rho}_{k} \frac{\rho_{k}}{\rho_{j}} R_{n l}^{(k)}\left(r_{j 3}\right)\left\{Y_{\lambda}\left(\hat{\rho}_{k}\right)\right. \\
&\left.\otimes Y_{l}\left(\hat{r}_{j 3}\right)\right\}_{L M}^{*}\left(V_{j 3}+V_{j k}\right)\left\{Y_{\lambda^{\prime}}\left(\hat{\rho}_{j}\right)\right. \\
&\left.\otimes Y_{l^{\prime}}\left(\hat{r}_{k 3}\right)\right\}_{L M} R_{n^{\prime} l^{\prime}}^{(j)}\left(r_{k 3}\right) f_{\alpha^{\prime}}^{(j)}\left(\rho_{j}\right) .
\end{aligned}
$$

For brevity we have defined $\alpha \equiv n l \lambda$ and $\alpha^{\prime} \equiv n^{\prime} l^{\prime} \lambda^{\prime}$, and omit the conserved total angular-momentum label $L M$. The functions $f_{\alpha}^{(k)}\left(\rho_{k}\right)$ depend on the scalar argument, but Eq. (14) is not yet one-dimensional. We are using the Jacobi coordinates

$$
\begin{gathered}
\vec{\rho}_{j}=\vec{r}_{j 3}-\beta_{k} \vec{r}_{k 3}, \quad \vec{r}_{j 3}=\frac{1}{\gamma}\left(\beta_{k} \vec{\rho}_{k}+\vec{\rho}_{j}\right), \\
\vec{r}_{j k}=\frac{1}{\gamma}\left(\sigma_{j} \vec{\rho}_{j}-\sigma_{k} \vec{\rho}_{k}\right),
\end{gathered}
$$

with

$$
\beta_{k}=\frac{m_{k}}{1+m_{k}}, \quad \sigma_{k}=1-\beta_{k}, \quad \gamma=1-\beta_{k} \beta_{j}, \quad j \neq k=1,2 .
$$

This shows that the modulus of $\vec{\rho}_{j}$ depends on two vectors: $\vec{\rho}_{j}=\gamma \vec{r}_{j 3}-\beta_{k} \vec{\rho}_{k}$. The integration in the right-hand side of Eq. (14) is done over these two vectors.

To obtain one-dimensional integral differential equations, corresponding to Eq. (14), we proceed with the integration over variables $\left\{\overrightarrow{\boldsymbol{\rho}}_{j}, \vec{\rho}_{k}\right\}$, rather than $\left\{\vec{r}_{j 3}, \vec{\rho}_{k}\right\}$. The Jacobian of this transformation is $\gamma^{-3}$. Thus, we come to a set of onedimensional integral differential equations

$$
\begin{gathered}
2 M_{k}\left(E-E_{n}^{(j)}\right) f_{\alpha}^{(k)}\left(\rho_{k}\right)+\left\{\frac{\partial^{2}}{\partial \rho_{k}^{2}}-\frac{\lambda(\lambda+1)}{\rho_{k}^{2}}\right\} f_{\alpha}^{(k)}\left(\rho_{k}\right) \\
=\frac{M_{k}}{\gamma^{3}} \sum_{\alpha^{\prime}} \int_{0}^{\infty} d \rho_{j} S_{\alpha \alpha^{\prime}}^{(k j)}\left(\rho_{k}, \rho_{j}\right) f_{\alpha^{\prime}}^{(j)}\left(\rho_{j}\right),
\end{gathered}
$$

where functions $S_{\alpha \alpha^{\prime}}^{(k j)}\left(\rho_{k}, \rho_{j}\right)$ are defined as follows:

$$
\begin{aligned}
S_{\alpha \alpha^{\prime}}^{(k j)}\left(\rho_{k}, \rho_{j}\right)= & 2 \rho_{k} \rho_{j} \int d \hat{\rho}_{j} \int d \hat{\rho}_{k} R_{n l}^{(k)}\left(r_{j 3}\right)\left\{Y_{\lambda}\left(\hat{\rho}_{k}\right)\right. \\
& \left.\otimes Y_{l}\left(\hat{r}_{j 3}\right)\right\}_{L M}^{*}\left(V_{j 3}+V_{j k}\right)\left\{Y_{\lambda^{\prime}}\left(\hat{\rho}_{j}\right)\right. \\
& \left.\otimes Y_{l^{\prime}}\left(\hat{r}_{k 3}\right)\right\}_{L M} R_{n^{\prime} l^{\prime}}^{(j)}\left(r_{k 3}\right) .
\end{aligned}
$$

The fourfold multiple integration in Eqs. (18) leads to a singlefold integral and the expression (18) for any value orbital momentum $L$ becomes

$$
\begin{aligned}
S_{\alpha \alpha^{\prime}}^{(k j)}\left(\rho_{k}, \rho_{j}\right)= & \frac{4 \pi}{2 L+1}\left[(2 \lambda+1)\left(2 \lambda^{\prime}+1\right)\right]^{1 / 2} \rho_{k} \rho_{j} \\
& \times \int_{0}^{\pi} d \omega \sin \omega R_{n l}^{(k)}\left(r_{j 3}\right)\left[V_{j 3}\left(r_{j 3}\right)+V_{j k}\left(r_{j k}\right)\right] \\
& \times R_{n^{\prime} l^{\prime}}^{(j)}\left(r_{k 3}\right) \sum_{m m^{\prime}} D_{m m^{\prime}}^{L}(0, \omega, 0) C_{\lambda 0 l m}^{L m} C_{\lambda^{\prime} 0 l^{\prime} m^{\prime}}^{L m^{\prime}} \\
& \times Y_{l m}\left(\nu_{j}, \pi\right) Y_{l^{\prime} m^{\prime}}^{*}\left(\nu_{k}, \pi\right),
\end{aligned}
$$

where $D_{m m^{\prime}}^{L}(0, \omega, 0)$ are Wigner functions, $\omega$ is the angle between $\vec{\rho}_{j}$ and $\vec{\rho}_{k}, \nu_{j}$ the angle between $\vec{r}_{k 3}$ and $\vec{\rho}_{j}$, and $\nu_{k}$ the angle between $\vec{r}_{j 3}$ and $\vec{\rho}_{k}$.

Finally, the set of integro-differential equations for the unknown functions $f_{n l \lambda}^{(k)}\left(\rho_{k}\right)$ can be written as

$$
\begin{aligned}
& {\left[\left(k_{n}^{(i)}\right)^{2}+\frac{\partial^{2}}{\partial \rho_{i}^{2}}-\frac{\lambda(\lambda+1)}{\rho_{i}^{2}}\right] f_{\alpha}^{(i)}\left(\rho_{i}\right)} \\
& =g_{i} \sum_{\alpha^{\prime}} \frac{\sqrt{(2 \lambda+1)\left(2 \lambda^{\prime}+1\right)}}{2 L+1} \int_{0}^{\infty} d \rho_{i^{\prime}} f_{\alpha^{\prime}}^{\left(i^{\prime}\right)}\left(\rho_{i^{\prime}}\right) \\
& \quad \times \int_{0}^{\pi} d \omega \sin \omega R_{n l}^{(i)}\left(\left|\vec{r}_{i^{\prime} 3}\right|\right)\left[-\frac{1}{\left|\vec{r}_{i^{\prime} 3}\right|}+\frac{1}{\left|\vec{r}_{i i^{\prime}}\right|}\right] \\
& \quad \times R_{n^{\prime} l^{\prime}}^{\left(i^{\prime}\right)}\left(\left|\vec{r}_{i 3}\right|\right) \rho_{i} \rho_{i^{\prime}} \sum_{m m^{\prime}} D_{m m^{\prime}}^{L}(0, \omega, 0)
\end{aligned}
$$


TABLE II. Cross sections $\sigma\left(\mathrm{D}-\mathrm{H}_{\mu}\right)=\sigma_{\mathrm{tr}} / 10^{-20} \mathrm{~cm}^{2}$ and rates $\lambda\left(\mathrm{D}-\mathrm{H}_{\mu}\right)=\lambda_{\mathrm{tr}} / 10^{10} \mathrm{~s}^{-1}$ for the $\mu$-transfer reaction $\mathrm{D}+\mathrm{H}_{\mu} \rightarrow \mathrm{D}_{\mu}+\mathrm{H}$, at different energies.

\begin{tabular}{|c|c|c|c|c|c|c|}
\hline \multirow{3}{*}{$\frac{E(\mathrm{eV})}{0.001}$} & $\sigma\left(\mathrm{D}-\mathrm{H}_{\mu}\right)$ & $\lambda\left(\mathrm{D}-\mathrm{H}_{\mu}\right)$ & $\sigma\left(\mathrm{D}-\mathrm{H}_{\mu}\right)$ & $\lambda\left(\mathrm{D}-\mathrm{H}_{\mu}\right)$ & $\sigma\left(\mathrm{D}-\mathrm{H}_{\mu}\right)$ & $\lambda\left(\mathrm{D}-\mathrm{H}_{\mu}\right)$ \\
\hline & \multicolumn{2}{|c|}{$1 s$} & \multicolumn{2}{|c|}{$1 s+2 s$} & \multicolumn{2}{|c|}{$1 s+2 s+2 p$} \\
\hline & 292.6 & 0.64 & 412.8 & 0.91 & 604.8 & 1.33 \\
\hline 0.01 & 92.3 & 0.64 & 130.0 & 0.90 & 190.0 & 1.32 \\
\hline 0.04 & 46.0 & 0.64 & 64.7 & 0.90 & 94.3 & 1.31 \\
\hline 0.1 & 29.0 & 0.64 & 40.8 & 0.90 & 59.4 & 1.31 \\
\hline 1.0 & 9.0 & 0.63 & 12.8 & 0.90 & 19.4 & 1.30 \\
\hline
\end{tabular}

$$
C_{\lambda 0 l m}^{L m} C_{\lambda^{\prime} 0 l^{\prime} m^{\prime}}^{L m^{\prime}} Y_{l m}^{*}\left(\nu_{i}, \pi\right) Y_{l^{\prime} m^{\prime}}\left(\nu_{i^{\prime}}, \pi\right)
$$

Here

$$
i \neq i^{\prime}=1,2, \quad g_{i}=4 \pi M_{i} / \gamma^{3}, \quad k_{n}^{(i)}=\sqrt{2 M_{i}\left(E-E_{n}^{\left(i^{\prime}\right)}\right)},
$$

$\omega$ is the angle between the Jacobi coordinates $\vec{\rho}_{i}$ and $\vec{\rho}_{i^{\prime}}, \quad \nu_{i}$ is the angle between $\vec{r}_{i^{\prime} 3}$ and $\vec{\rho}_{i}$ and $\nu_{i^{\prime}}$ is the angle between $\vec{r}_{i 3}$ and $\vec{\rho}_{i}$, with

$$
\sin \nu_{i}=\frac{\rho_{i^{\prime}}}{\gamma r_{i^{\prime} 3}} \sin \omega, \quad \text { and } \quad \cos \nu_{i}=\frac{1}{\gamma r_{i^{\prime} 3}}\left(\beta_{i} \rho_{i}+\rho_{i^{\prime}} \cos \omega\right)
$$

To find a unique solution to system (20), appropriate boundary conditions need to be considered. First we impose $f_{n l}^{(i)}(0)=0$. For the present scattering problem with $1+(23)$ as the initial state, in the asymptotic region two solutions to Eq. (20) satisfy the following boundary conditions:

$$
\begin{gathered}
f_{1 s}^{(1)}\left(\rho_{1}\right) \underset{\rho_{1} \rightarrow+\infty}{\longrightarrow} \sin \left(k_{1}^{(1)} \rho_{1}\right)+K_{11} \cos \left(k_{1}^{(1)} \rho_{1}\right), \\
f_{1 s}^{(2)}\left(\rho_{2}\right) \widetilde{\rho_{2} \rightarrow+\infty} \sqrt{\mathrm{v}_{1} / \mathrm{v}_{2}} K_{12} \cos \left(k_{1}^{(2)} \rho_{2}\right),
\end{gathered}
$$

where 1 refers to channel $1+(23), 2$ to channel $2+(13)$ and $K$ denotes the corresponding on-shell $\mathbf{K}$ matrix [28]. For scattering with $2+(13)$ as the initial state, we have the following conditions:

$$
\begin{gathered}
f_{1 s}^{(1)}\left(\rho_{1}\right) \widetilde{\rho_{1} \rightarrow+\infty} \sqrt{\mathrm{v}_{2} / \mathrm{v}_{1}} K_{21} \cos \left(k_{1}^{(1)} \rho_{1}\right), \\
f_{1 s}^{(2)}\left(\rho_{2}\right) \underset{\rho_{2} \rightarrow+\infty}{\longrightarrow} \sin \left(k_{1}^{(2)} \rho_{2}\right)+K_{22} \cos \left(k_{1}^{(2)} \rho_{2}\right),
\end{gathered}
$$

where $\mathrm{v}_{i}, \quad i=1,2$ are velocities in channel $i$. With the following change of variables in Eqs. (20):

$$
\begin{aligned}
& \mathbf{f}_{1 s}^{(1)}\left(\rho_{1}\right)=f_{1 s}^{(1)}\left(\rho_{1}\right)-\sin \left(k_{1}^{(1)} \rho_{1}\right), \\
& \mathbf{f}_{1 s}^{(2)}\left(\rho_{2}\right)=f_{1 s}^{(2)}\left(\rho_{2}\right)-\sin \left(k_{1}^{(2)} \rho_{2}\right),
\end{aligned}
$$

we can obtain two sets of inhomogeneous equations that are solved numerically. The cross sections are given by

$$
\sigma_{i j}=\frac{4 \pi}{k_{1}^{(i) 2}}\left|\frac{\mathbf{K}}{1-i \mathbf{K}}\right|^{2}=\frac{4 \pi}{k_{1}^{(i) 2}} \frac{\delta_{i j} D^{2}+K_{i j}^{2}}{(D-1)^{2}+\left(K_{11}+K_{22}\right)^{2}},
$$

where $i=j=1,2$ refer to the two channels and

$$
D=\operatorname{det} \mathbf{K}=K_{11} K_{22}-K_{12} K_{21} .
$$

When $k_{1}^{(1)} \rightarrow 0, \quad K_{12}=K_{21} \sim k_{1}^{(1)}, \quad K_{11} \sim k_{1}^{(1)}$; in this case $\sigma_{\mathrm{tr}} \equiv \sigma_{12} \sim 1 / k_{1}^{(1)}$ and $\sigma_{\mathrm{el}}=\sigma_{11} \sim$ const. For comparison with experimental low-energy data it is very useful to calculate the transfer rates $(2)$ because $\lambda_{\text {tr }}\left(k_{1}^{(1)} \rightarrow 0\right) \sim$ const.

\section{NUMERICAL RESULTS}

To solve the integro-differential equation, one has to calculate the angle integrals in Eq. (20) that are independent of the energy $E$. One needs to calculate them only once and store them on a hard disk for the calculation of other observables; for instance, the cross sections at different energies. Subintegrals in Eq. (20) have a strong dependence on $\rho_{i}$ and $\rho_{i^{\prime}} \quad\left(i \neq i^{\prime}=1,2\right)$. To calculate $S_{\alpha \alpha^{\prime}}^{\left(i i^{\prime}\right)}\left(\rho_{i}, \rho_{i^{\prime}}\right)$ at different coordinates an adaptable algorithm has been used. In this case, using the relation

$$
\cos \omega=\frac{x^{2}-\beta_{i}^{2} \rho_{i}^{2}-\rho_{i^{\prime}}^{2}}{2 \beta_{i} \rho_{i} \rho_{i^{\prime}}},
$$

the angle-dependent part of Eq. (20) can be written as the following integral:

$$
\begin{aligned}
S_{\alpha \alpha^{\prime}}^{\left(i i^{\prime}\right)}\left(\rho_{i}, \rho_{i^{\prime}}\right)= & \frac{4 \pi}{\beta_{i}} \frac{\left[(2 \lambda+1)\left(2 \lambda^{\prime}+1\right)\right]^{1 / 2}}{2 L+1} \\
& \times \int_{\left|\beta_{i} \rho_{i}-\rho_{i^{\prime}}\right|}^{\beta_{i} \rho_{i}+\rho_{i^{\prime}}} d x R_{n l}^{(i)}(x)\left[-1+\frac{x}{r_{i i^{\prime}}(x)}\right] \\
& \times R_{n^{\prime} l^{\prime}}^{\left(i^{\prime}\right)}\left(r_{i 3}(x)\right) \sum_{m m^{\prime}} D_{m m^{\prime}}^{L}(0, \omega(x), 0) C_{\lambda 0 l m}^{L m} \\
& \times C_{\lambda^{\prime} 0 l^{\prime} m^{\prime}}^{L m^{\prime}} Y_{l m}^{*}\left(\nu_{i}(x), \pi\right) Y_{l^{\prime} m^{\prime}}\left(\nu_{i^{\prime}}(x), \pi\right) .
\end{aligned}
$$

Note that the expression (28) differs from zero only in a narrow strip when $\rho_{i} \approx \rho_{i^{\prime}}$. 
TABLE III. Cross sections $\sigma\left(\mathrm{T}-\mathrm{H}_{\mu}\right)=\sigma_{\mathrm{tr}} / 10^{-20} \mathrm{~cm}^{2}$ and rates $\lambda\left(\mathrm{T}-\mathrm{H}_{\mu}\right)=\lambda_{\mathrm{tr}} / 10^{10} \mathrm{~s}^{-1}$ for the $\mu$-transfer reaction $\mathrm{T}+\mathrm{H}_{\mu} \rightarrow \mathrm{T}_{\mu}+\mathrm{H}$, at different energies.

\begin{tabular}{lcccccc}
\hline \hline & $\sigma\left(\mathrm{T}-\mathrm{H}_{\mu}\right)$ & $\lambda\left(\mathrm{T}-\mathrm{H}_{\mu}\right)$ & $\sigma\left(\mathrm{T}-\mathrm{H}_{\mu}\right)$ & $\lambda\left(\mathrm{T}-\mathrm{H}_{\mu}\right)$ & $\sigma\left(\mathrm{T}-\mathrm{H}_{\mu}\right)$ & \multicolumn{2}{c}{$\lambda\left(\mathrm{T}-\mathrm{H}_{\mu}\right)$} \\
$E(\mathrm{eV})$ & & $1 s$ & \multicolumn{2}{c}{$1 s+2 s$} & \multicolumn{2}{c}{$1 s+2 s+2 p$} \\
\hline 0.001 & 204.2 & 0.42 & 249.4 & 0.52 & 294.4 & 0.61 \\
0.01 & 64.3 & 0.42 & 78.5 & 0.51 & 92.6 & 0.60 \\
0.04 & 31.9 & 0.42 & 38.9 & 0.51 & 45.8 & 0.60 \\
0.1 & 19.9 & 0.41 & 24.3 & 0.50 & 28.6 & 0.60 \\
1.0 & 5.50 & 0.36 & 6.70 & 0.44 & 8.0 & 0.52 \\
\hline \hline
\end{tabular}

We employ a muonic atomic unit: distances are measured in units of $a_{\mu}$, where $a_{\mu}$ is the radius of the muonic hydrogen atom. The integro-differential equations were solved by the usual numerical procedure by discretizing them into a linear system of equations, which are subsequently solved by the Gauss elimination method. In solving these equations, distances up to $50 a_{\mu}$ were considered and 400-600 points were used in the discretization. The following mass values are used in the unit of electron mass: $m_{H}=1836.152, m_{D}$ $=3670.481$, and $m_{T}=5496.918$, and the muon mass is $m_{\mu}$ $=206.769$.

Tables II, III, and IV include our results for the muonic transfer cross sections and rates for all hydrogen isotopes (1) using different approximation schemes. We present results for the two-, four-, and six-state approximations where we include $1 s, 1 s+2 s$, and $1 s+2 s+2 p$ states of the muonic atoms in the initial and final channels, respectively. In solving the equations, we employed only the lowest partial wave, e.g., $L=0$. As we shall mainly be concerned with the experimental muon transfer rates at very low energies, the higher partial waves are expected to have a negligible contribution. The $2 p$ states are found to contribute significantly in $\mathrm{T}-\mathrm{D}_{\mu}$, moderately in $\mathrm{D}-\mathrm{H}_{\mu}$, and little in $\mathrm{T}-\mathrm{H}_{\mu}$ systems. This is in agreement with a similar conclusion in Ref. [24] for the $\mathrm{T}-\mathrm{D}_{\mu}$ system. This could be understood qualitatively from the following consideration. At zero incident energy the relative velocity in the final state after muon transfer is the highest in the case of $\mathrm{T}-\mathrm{H}_{\mu}$, lowest in the case of $\mathrm{T}-\mathrm{D}_{\mu}$, and intermediate in the case of $\mathrm{D}-\mathrm{H}_{\mu}$. It is expected that the polarization potential arising out of a $1 s+2 s+2 p$ calculation will have the largest effect on convergence when the final-state velocity is the lowest. Hence the necessity of the higher-order states is more pronounced in the case of $\mathrm{T}-\mathrm{D}_{\mu}$ and less pronounced in the case of $\mathrm{T}-\mathrm{H}_{\mu}$. We also find that as energy decreases the transfer cross sections increase and the transfer rates attain a constant value. These transfer rates are essentially constant below $0.1 \mathrm{eV}$ and are also measured experimentally, so that we can compare our rates with other experimental and theoretical results.

For the D- $\mathrm{H}_{\mu}$ system the present low-energy muon transfer rate of $133 \times 10^{8} \mathrm{~s}^{-1}$ is in agreement with both experiments $[13,14]$. The present rate is slightly smaller than the theoretical studies of Refs. [20] and [21] and this makes the agreement with experiment better. For the $\mathrm{T}-\mathrm{H}_{\mu}$ system again the present result of $61 \times 10^{8} \mathrm{~s}^{-1}$ is in better agreement with experiment [16] than the other theoretical studies. In case of $T-D_{\mu}$, the present result $2.3 \times 10^{8} \mathrm{~s}^{-1}$ is also in very good agreement with experiment.

Within the six-state approximation our cross sections for low-energy elastic scattering in the case of the $\mathrm{T}-\mathrm{D}_{\mu}$ system are presented in Table $\mathrm{V}$ together with other theoretical results. The present cross sections attain a constant value at low energies and are in fairly good agreement with the results of other studies.

As a futher test of the present few-body approach, we have also calculated $S$-wave cross sections of antihydrogen formation in antiproton-positronium low-energy collisions (3). In Table VI our results within the six-state approximation $(\mathrm{Ps}[1 s+2 s+2 p], \overline{\mathrm{H}}[1 s+2 s+2 p])$ are compared with calculations based on the hyperspherical coupled-channel method [26]. Considering that the present calculation is limited to only the lowest partial wave $(L=0)$ and to a truncated basis set $(1 s+2 s+2 p)$, the agreement is reasonable for energies below $1 \mathrm{eV}$. However, at $2 \mathrm{eV}$ the agreement is not so good. The reason for this is not clear at present. Further theoretical investigation including higher partial waves with an extended basis set could reveal the trend of the converged cross sections.

TABLE IV. Cross sections $\sigma\left(\mathrm{T}-\mathrm{D}_{\mu}\right)=\sigma_{\mathrm{tr}} / 10^{-20} \mathrm{~cm}^{2}$ and rates $\lambda\left(\mathrm{T}-\mathrm{D}_{\mu}\right)=\lambda_{\mathrm{tr}} / 10^{8} \mathrm{~s}^{-1}$ for the $\mu$-transfer reaction $\mathrm{T}+\mathrm{D}_{\mu} \rightarrow \mathrm{T}_{\mu}+\mathrm{D}$, at different energies.

\begin{tabular}{lcccccc}
\hline \hline & $\sigma\left(\mathrm{T}_{-} \mathrm{D}_{\mu}\right)$ & $\lambda\left(\mathrm{T}-\mathrm{D}_{\mu}\right)$ & $\sigma\left(\mathrm{T}-\mathrm{D}_{\mu}\right)$ & $\lambda\left(\mathrm{T}_{-} \mathrm{D}_{\mu}\right)$ & $\sigma\left(\mathrm{T}_{-} \mathrm{D}_{\mu}\right)$ & \multicolumn{2}{c}{$\lambda\left(\mathrm{T}-\mathrm{D}_{\mu}\right)$} \\
\multicolumn{1}{c}{$E(\mathrm{eV})$} & & $1 s$ & \multicolumn{2}{c}{$1 s+2 s$} & \multicolumn{2}{c}{$1 s+2 s+2 p$} \\
\hline 0.001 & 4.58 & 0.77 & 5.05 & 0.84 & 13.7 & 2.3 \\
0.01 & 1.44 & 0.76 & 1.60 & 0.84 & 4.3 & 2.3 \\
0.04 & 0.71 & 0.75 & 0.78 & 0.83 & 2.14 & 2.26 \\
0.1 & 0.44 & 0.73 & 0.48 & 0.81 & 1.32 & 2.21 \\
1.0 & 0.1 & 0.44 & 0.1 & 0.5 & 0.3 & 1.5 \\
\hline \hline
\end{tabular}


TABLE V. Elastic cross sections for $\mathrm{T}^{-\mathrm{D}_{\mu}}$ collision in units of $10^{-20} \mathrm{~cm}^{2}$ at different energies.

\begin{tabular}{lcccc}
\hline \hline$E(\mathrm{eV})$ & $\begin{array}{c}\text { Present results } \\
1 s+2 s+2 p\end{array}$ & {$[22]$} & {$[24]$} & {$[25]$} \\
\hline 0.001 & 1.2 & 1.7 & 1.63 & 2.014 \\
0.01 & 1.3 & 2.3 & 2.15 & 3.605 \\
\hline \hline
\end{tabular}

\section{CONCLUSION}

The study of three-body Coulombic systems has been the subject of this work. We have formulated a method for a few-body description of the rearrangement scattering problem by solving the Faddeev-Hahn-type equations in coordinate space. It is shown that within this formalism the application of a close-coupling-type ansatz leads to satisfactory results already in low-order approximations for (i) muontransfer reactions between hydrogen isotopes and (ii) antihydrogen formation in the antiproton-positronium collision. Because of computational difficulties, in this preliminary application we have considered up to six states in the expansion scheme $(1 s+2 s+2 p$ on each center), which may not always be adequate. Further calculations with larger basis sets are needed to obtain the converged results.

The present model leads to a reduction of the usual technical effort and is definitely worth using for investigations of larger systems. It seems reasonable to suppose that the method should be an effective tool for the description of other muonic and atomic few-body collisions. For instance, one could study using the present approach the following muon-transfer reactions to elements with $Z \geqslant 2$ :

$$
\left(\mathrm{H}_{\mu}\right)_{1 s}+\mathrm{X}^{Z} \rightarrow \mathrm{X}_{\mu}^{Z}+\mathrm{H}
$$

TABLE VI. Cross sections in unit of $\pi a_{0}^{2}$ for the positron transfer reaction $\bar{p}+\mathrm{Ps} \rightarrow \overline{\mathrm{H}}+e^{-}$.

\begin{tabular}{|c|c|c|c|c|c|}
\hline$E(\mathrm{eV})$ & $\begin{array}{l}\text { Present results } \\
1 s+2 s+2 p\end{array}$ & [26] & $E(\mathrm{eV})$ & $\begin{array}{l}\text { Present results } \\
1 s+2 s+2 p\end{array}$ & {$[26]$} \\
\hline 0.1 & 1.5 & $2.3^{\mathrm{a}}$ & 1.0 & 3.2 & $3.5^{\mathrm{a}}$ \\
\hline 0.5 & 2.0 & $2.6^{\mathrm{a}}$ & 2.0 & 1.7 & $3.7^{\mathrm{a}}$ \\
\hline
\end{tabular}

${ }^{a}$ The cross sections estimated from Fig. 1 of Ref. [26].

where the cross section depends in a complicated manner on the charge $Z[5]$.

Theoretically, the reaction (29) is of much interest as an example of low-energy rearrangement scattering in a system of three charged particles with Coulomb repulsion in the final state. Evidently it makes additional difficulties for a correct theoretical description of Eq. (29) [12]. The FaddeevHahn-type approach seems to be suitable for the study of such reactions and would be a topic of future investigation. We are presently in the process of studying reaction (29) with the present method for $Z=2$ and 3 . We also plan to employ an extended basis set with more basis functions in the future. Also, the excited-state muon-transfer reactions of recent experimantal and theoretical interest [29,30] could be studied with the present model.

\section{ACKNOWLEDGMENTS}

We acknowledge the support from FAPESP (Fundação de Amparo ã Pesquisa do Estado de São Paulo) of Brazil. The numerical calculations have been performed on the IBM SP2 Supercomputer of the Departamento de Física-IBILCEUNESP, São José do Rio Preto, Brazil.
[1] S. Tresch, F. Mulhauser, C. Piller, L. A. Schaller, L. Schellenberg, H. Schneuwly, Y. A. Thalmann, A. Werthmuller, P. Ackerbauer, W. H. Breunlich, M. Cargnelli, B. Gartner, R. King, B. Lauss, J. Marton, W. Prymas, J. Zmeskal, C. Petitjean, M. Augsburger, D. Chatellard, J. P. Egger, E. Jeannet, T. von Egidy, F. J. Hartmann, M. Muhlbauer, and W. Schott, Phys. Rev. A 58, 3528 (1998).

[2] S. Tresch, R. Jacot-Guillarmod, F. Mulhauser, C. Piller, L. A. Schaller, L. Schellenberg, H. Schneuwly, Y. A. Thalmann, A. Werthmuller, P. Ackerbauer, W. H. Breunlich, M. Cargnelli, B. Gartner, R. King, B. Lauss, J. Marton, W. Prymas, J. Zmeskal, C. Petitjean, D. Chatellard, J. P. Egger, E. Jeannet, F. J. Hartmann, and M. Muhlbauer, Phys. Rev. A 57, 2496 (1998).

[3] Y.-A. Thalmann, R. Jacot-Guillarmod, F. Mulhauser, L. A. Schaller, L. Schellenberg, H. Schneuwly, S. Tresch, and A. Wertmüller, Phys. Rev. A 57, 1713 (1998).

[4] S. Tresch, R. Jacot-Guillarmod, F. Mulhauser, L. A. Schaller, L. Schellenberg, H. Schneuwly, Y.-A. Thalmann, and A. Werthmüller, Eur. Phys. J. D 2, 93 (1998).

[5] F. Mulhauser and H. Schneuwly, J. Phys. B 26, 4307 (1993);
L. Schellenberg, Hyperfine Interact. 82, 513 (1993).

[6] W. H. Breunlich, P. Kammel, J. S. Cohen, and M. Leon, Annu. Rev. Nucl. Part. Sci. 39, 311 (1989).

[7] M. H. Holzscheiter, G. Bendiscioli, A. Bertin, G. Bollen, M. Bruschi, C. Cesar, M. Charlton, M. Corradini, D. DePedis, M. Doser, J. Eades, R. Fedele, X. Feng, F. Galluccio, T. Goldman, J. S. Hangst, R. Hayano, D. Horváth, R. J. Hughes, N. S. P. King, K. Kirsebom, H. Knudsen, V. Lagomarsino, R. Landua, G. Laricchia, R. A. Lewis, E. LodiRizzini, M. Macri, G. Manuzio, U. Marconi, M. R. Masullo, J. P. Merrison, S. P. Moller, G. L. Morgan, M. M. Nieto, M. Piccinini, R. Poggiani, A. Rotondi, G. Rouleau, P. Salvini, N. Semprini, N. Cesari, G. A. Smith, C. M. Surko, G. Testera, G. Torelli, E. Uggerhoj, V. G. Vaccaro, L. Venturelli, A. Vitale, E. Widmann, T. Yamazaki, Y. Yamazaki, D. Zanello, and A. Zoccoli, Hyperfine Interact. 109, 1 (1997).

[8] J. Eades and F. J. Hartmann, Rev. Mod. Phys. 71, 373 (1999); M. Charlton, J. Eades, D. Horváth, R. J. Hughes, and C. Zimmerman, Phys. Rep. 241, 65 (1994).

[9] Y. Hahn, Phys. Rev. 169, 794 (1968).

[10] Y. Hahn and K. M. Watson, Phys. Rev. A 5, 1718 (1972). 
[11] L. D. Faddeev, Zh. Éksp. Teor. Fiz. 39, 1459 (1960) [Sov. Phys. JETP 12, 1014 (1961)].

[12] R. A. Sultanov, W. Sandhas, and V. B. Belyaev, Eur. Phys. J. D 5, 33 (1999).

[13] V. P. Dzhelepov, P. F. Ermolov, E. A. Kushnirenko, V. I. Moskalev, and S. S. Gershtein, Zh. Éksp. Theor. Fiz. 42, 439 (1962) [Sov. Phys. JETP 15, 306 (1962)].

[14] E. J. Bleser, E. W. Anderson, L. M. Lederman, S. L. Meyer, J. L. Rosen, J. E. Rothberg, and I-T. Wang, Phys. Rev. 132, 2679 (1963).

[15] A. Bertin, M. Bruno, V. Vitale, A. Placci, and E. Zavattini, Lett. Nuovo Cimento 4, 449 (1972).

[16] F. Mulhauser, J. L. Beveridge, G. M. Marshall, J. M. Bailey, G. A. Beer, P. E. Knowles, G. R. Mason, A. Olin, M. C. Fujiwara, T. M. Huber, R. Jacot-Guillarmod, P. Kammel, J. Zmeskal, S. K. Kim, A. R. Kunselman, V. E. Markushin, C. J. Martoff, and C. Petitjean, Phys. Rev. A 53, 3069 (1996).

[17] V. M. Bystritsky, V. P. Dzhelepov, Z. V. Yershova, V. G. Zinov, V. K. Kapyshev, S. S. Mukhametgaleyeva, V. S. Nadezhdin, L. A. Rivkis, A. I. Rudenko, V. I. Satarov, N. V. Sergeyeva, L. N. Somov, V. A. Stolupin, and V. V. Filchenkov, Zh. Éksp.Teor. Fiz. 80, 1700 (1980) [Sov. Phys. JETP 53, 877 (1981)].

[18] S. E. Jones, A. N. Anderson, A. J. Caffrey, J. B. Walter, K. D. Watts, J. N. Bradbury, M. Leon, H. R. Maltrud, and M. A. Paciotti, Phys. Rev. Lett. 51, 1757 (1983).

[19] W. H. Breunlich, M. Cargnelli, P. Kammel, J. Marton, N. Nae- gele, P. Pawlek, A. Scrinzi, J. Werner, J. Zmeskal, J. Bistirlich, K. M. Crowe, M. Justice, J. Kurck, C. Petitjean, R. H. Sherman, H. Bossy, H. Daniel, F. J. Hartmann, W. Neumann, and G. Schmidt, Phys. Rev. Lett. 58, 329 (1987).

[20] J. S. Cohen and M. C. Struensee, Phys. Rev. A 43, 3460 (1991).

[21] A. Adamczak, C. Chiccoli, V. I. Korobov, V. S. Melezhik, P. Pasini, L. I. Ponomarev, and J. Wozniak, Phys. Lett. B 285, 319 (1992).

[22] H. Fukuda, T. Ishihara, and S. Hara, Phys. Rev. A 41, 145 (1990).

[23] Y. Kino and M. Kamimura, Hyperfine Interact. 82, 45 (1993).

[24] A. Igarashi, N. Toshima, and T. Shirai, Phys. Rev. A 50, 4951 (1994).

[25] A. Boukour, R. N. Hewitt, and Ch. Leclercq-Willain, J. Phys. B 29, 4309 (1996).

[26] A. Igarashi, N. Toshima, and T. Shirai, J. Phys. B 27, L497 (1994).

[27] J. Mitroy and G. Ryzhikh, J. Phys. B 30, L371 (1997).

[28] N. F. Mott and H. S. W. Massey, The Theory of Atomic Collisions (Clarendon, London, 1965).

[29] B. Lauss, P. Ackerbauer, W. H. Breunlich, B. Gartner, M. Jeitler, P. Kammel, J. Marton, W. Prymas, J. Zmeskal, D. Chatellard, J. P. Egger, E. Jeannet, H. Daniel, A. Kosak, F. J. Hartmann, and C. Petitjean, Phys. Rev. Lett. 76, 4693 (1996).

[30] A. V. Kravtsov and A. I. Mikhailov, Phys. Rev. A 58, 4426 (1998). 